\title{
VII. On the optical character of some brilliant animal colours
}

\section{Lord Rayleigh O.M. F.R.S.}

To cite this article: Lord Rayleigh O.M. F.R.S. (1919) VII. On the optical character of some brilliant animal colours, Philosophical Magazine Series 6, 37:217, 98-111, DOI: 10.1080/14786440108635867

To link to this article: http://dx.doi.org/10.1080/14786440108635867

曲 Published online: 08 Apr 2009.

Submit your article to this journal $\lceil\pi$

山 Article views: 13

Q View related articles $\square$

4 Citing articles: 29 View citing articles ¿ 
VII. On the Optical Character of some Brilliant Animal Colours. By Lord RAYLEIGH, O.M., F.R.S.*

T $T$ is singular that the explanation of some of the most 1 striking and beautiful of optical phenomena should be still matters of controversy. I allude to the brilliant colours displayed by many birds (e. g. humming-birds), butterflies, and beetles, colours which vary greatly with the incidence of the light, and so cannot well be referred to the ordinary operation of dyes. In an early paper $\dagger$, being occupied at the time with the remarkable coloured reflexions from certain crystals of chlorate of potash described by Stokes, and which $I$ attributed to a periodic twinning $\ddagger$, I accepted, perhaps too hastily, the view generally current among naturalists that these colours were "structure-colours," more or less like those of thin plates, as in the soap-brbble. Among the supporters of this view $\$$ in more recent times may be especially mentioned Poulton and Hodgkinson. In Poulton's paper $\|$ the main purpose was to examine the history of the very remarkable connexion between the metallic colours of certain pupæ (especially Vanessa urticce) and the character of the light to which the larvæ are exposed before pupation. In a passage describing the metallic colour itself he remarks :-

"The Nature of the Effects Produced.-The gilded appearance is one of the most metal-like appearances in any non-metallic substance. The optical explanation has never been understood. It has, however, been long known that it depends upon the cuticle, and needs the presence of moisture, and that it can be renewed when the dry cuticle is moistened. Hence it can be preserved for any time in spirit. If a piece of dry cuticle be moistened on its upper surface the colour is not renewed, but almost instantly follows the application of spirit to the lower surface. Sections of the cuticle resemble those of Papilio machaon described in a previous paper (Roy. Soc. Proc. vol. xxxviii. p. 279, 1885), and show an upper thin layer and a lower, much thicker, finely laminated layer which is also striated vertically to the surface. With Prof. Clifton's kind assistance I have been

* Communicated by the Author.

† Phil. Mag. vol. xxiv. p. 145 (1887); Scientific Papers, vol. iij. p. 13, see footnote.

$\ddagger$ Phil, Mag. vol. xxvi. p. 256 (1888); Scientific Papers, vol iii. p. 204.

\$ Distinctly suggested by Hooke in his 'Micrographia' (1665).

if Roy. Soc. Proc. vol. xlii. p. 94 (1887). 
able to show that the appearances follow from interference of light, due to the presence of films of liquid between the limella of the lower layer. The microscope shows brilliant red and green tints by reflected light, while in transmitted light the complementary colours are distinct, but without hrilliancy. The latter colours are seen to change when pressure is applied to the surface of the cuticle, and when the process of drying is watched under the microscope, owing in both cases to the liquid films becoming thinner. In the dry cuticle the solid lamellæ probably come into contact, and prevent the admission of air, wbich, if present, would cause even greater brilliancy than liquid. The spectroscope shows broad interference-bands in the transmitted light, which change their position on altering the angle of incidence of the light which passes through the cuticle. Precisely similar colours, metallic on reflexion, non-metaliic and with complementary tints on transmission, with the same spectroscopic appearances and changes induced by the same means, are seen in the surface films which are formed on bottle-ylass alter prolonged exposure to earth and moisture. In the alternating layers of the pupa the chitinous lamella are of ligher, the liquid films of lower refractive index; hence water or alcohol produces brilliant appearances, while liquids of higher refractive indices produce less effect."

I owe to Prof. Poulton the opportunity of repeating some of these observations, such as the loss of metallic appearance on drying and of recovery under alcohol. On substitution of benzol with a little bisulphide of carbon for alcohol, the surface became very dark, but regained the golden glitter on going back to alcohol.

Of a specimen of another kind Prof. Poulton writes that the bug has been in the Oxford Museum Collection for 30 or 40 years judged by the pin. It is brown when dry, but when soaked in water becomes green like a leaf with bright iridescent green stripes on the under side. This observation also I have been able to repeat. All of which, it need hardly be said, is strongly suggestive of interference.

Dr. A. Hodgkinson also has described interesting observations. In his early papers * he distinctly refers the colours to Newton's scale, which in strictness would imply a limitation to a single thin plate. He emphasises the importance. for purposes of identification, of recording the colours of feathers \&c. as seen by perpendicular reflexion, a condition best secured by illumination from a small perforated mirror,

* Manchester Nemoirs, 1889; 1892, p. 149.

$\mathrm{H} 2$ 
behind which the eye is placed. When daylight is used, it often suffices to examine the object with one's back to the window and at some distance from it. I shall have occasion later to refer agrain to Hodgkinson's work.

The first, so far as I know, to challenge the "structure" theory was Dr. B. Walter, whose tract* includes an elaborate discussion, accompanied by original observations, of the colours which may arise in the act of reflexion, and decides unequivocally that the colours now in question, with one or two possible exceptions, are due to surface, or quasi-metallic, reflexion as described by Hiaidinger, Brewster, and Stokes. The first of these writers formulated a law, named after him, which identifies the surface-colour with those rays which would be most intensely absorbed within the substance. The theory of " anomalous dispersion" since developed shows, however, that the matter does not stop there, and Walter emphasises that much of the surface-colour may be ascribed to rays which are not themselves intensely absorbed, but being situated near an absorption-band are abnormally refracted, and hence in accordance with Fresnel's laws are abnormally reflected. On the red side of the band the refractive index is increased and on the blue side diminished, so that when the substance is in air the surface reflexion is redder than according to Haidinger's law; but this conclusion may need to be modified when the substance is in contact with a strongly refractive solid, as when a dye spread upon a glass plate is examined from the glass side. In some cases it appears that the surface-colour is dne as much, or even more, to these rays excessively refracted (and consequently reflected) as to those which would be intensely absorbed and are reflected in accordance with Haidinger's rule.

The departure from Haidinger's rule is specially important when we consider what happens at oblique incidences and with polarized light. The rays reflected in virtue of the extreme opacity of the substance to them are comparatively unaffected, and are indeed rendered more prominent by the appropriate use of a nicol. As Stokes says $\dagger$ : "In the case of the substances at present considered, the reflected light does not vanish, but at a considerable angle of incidence the pencil polarized perpendicularly to the plane of incidence becomes usually of a richer colour, in consequence of the removal, in great measure, of that portion of the reflected light which is independent of the metallic properties of the

* Die Oberfächen oller schillerfarben, Braunschweig, 1895.

$\dagger$ Phil. Mag. vol. vi. Jec. 1853, p. 393; Math. and Phys. Papers, vol. iv. p. 42 . 
medium; it commonly becomes, also, more strictly related to that light which is absorbed with such great intensily." But, as Walter appears to have been the first to explain, there is a further important change of colour with the angle of incidence, when the light-vibrations are in the plane of incidence, in virtue of the abnormal refraction with its accompanying abnormal polarizing angle. In the usual case, where the dye is in contact with air, the polarizing angle for the rays lying on the red side of the absorptionband is unusually high, so that these rays, which at moderate angles of incidence contribute largely to the resultant colour, are extinguished at incidences of from $60^{\circ}$ to $70^{\circ}$. In consequence, the colour of the reflected light moves towards the blue with increasing obliquity.

As an example, fuchsin may be referred to, a dye specially studied by Walter, who thus (p. 52) describes the surfacecolour as seen from the air side :-

"(a) For light polarized in the plane of incidence :

"At small angles of incidence the reflexion is yellowgreen, and at increasing angles becomes ever yellower and brighter.

"(b) For light polarized perpendicularly to the plane of incidence (that is, vibrating in this plane) :

"At perpendicular incidence the reflexion is the same as under $(a)$, and remains approximately so up to incidences of $50^{\circ}$. At about $60^{\circ}$ it becomes rapidly blue-green and at $70^{\circ}$ an almost pure blue, attaining its greatest purity at about $72^{\circ}$. At still greater angles the colour passes rapidly into a bright violet, and at $85^{\circ}$ into white.

"When ordinary unpolarized light is employed, the colour of the reflexion is intermediate between $(a)$ and $(b)$, but always nearer to $(a)$ than to $(b)$, on account of the greater intensity of reflexion under $(a)$."

It is this movement of surface-reflexions towards the blue with increasing obliquity which is regarded by Walter and Michelson * as annulling the presumption in favour of the structure theory of the animal colours, which also move in this direction; and it must, of course, be admitted that the criterion is somewhat blurred thereby. Walter, indeed, maintains that thin plate colours change too much with angle to meet the requirements of the case. To this point I will return presently; but what I wish to remark at the moment is that with ordinary unpolarized light the surface-colours

* Phil. Mag. vol. xxi. p. 554 (1911). "On Metallic Colouring in Birds and Insects." 
appear to change too little. Neither in the case of fuchsin nor of diamond green $\mathrm{G}$ - the second dye specially discussed by Walter,-or with any other dye hitherto examined*, have I seen an adequate change of colour without the use of the nicol to eliminate vibrations in the plane perpendicular to that of incidence. In the absence of a nicol there is little sign of the blue seen with it from fuchsin at $70^{\circ}$ incidence. Much greater changes with more saturated colour are exlibited by the wing-cases of beetles when so examined.

As to the adequacy of the suriace-colours Michelson himself remarks :- "indeed, it may perhaps be objected that the (animal) colours are far more vivid than any of the reflexion hues of the aniline dyes, or of any other case of 'surfacecolour' hitherto observed." But perhaps this objection should not be very much insisted on in our ignorance of nature's operations and with regard to the known existence of powerful dyes, e.g. in feathers. It is rather the rapid loss of purity with obliquity in surface-colour which appears significant.

If a dye capable of surface-reflexion is present, there are still alternatives open. The pure or nearly pure dye may be on the outside so as to be in contact with air, or it may be overlaid by a colourless skin of horny material (chitin) in optical contact with it. The former case would be the more favourable for vivid and variable colour, but then one would expect to be able to remove the dye by solvents. So far as I am aware this has not been done, and my own trials with various solvents upon the wing-cases of beetles have not succeeded. The most satisfactory demonstration of the surface-colour theory would indeed be the extraction of the dye and its exhibition as a thin layer spread upon glass.

If, on the other hand, the dye is imprisoned within a layer of colourless chitin, the range of obliquities available in ordinary observation would be restricted and the difficulty of accounting for the variety of nearly saturated hues actually seen would be increased, more especially when we remember the dilution with white light reflected at the external surface.

* Throngh the lindness of Sir J. Dewar I have had the opportunity of experimenting with a gond many dyes from the Badische AnilinFabrik. Following Walter, I have used warm alcoholic solutions spread upon previously warmed glass plates. IJatterly I have exmmined some more dyes, for which I an indebted to Prof. Green. In no case have I seen any considerable change of well-developed colour unless the light was polarized. 
There is still another viow, which indeed is that actually maintained by Walter, whose argument and conclusion * it may be well to quote :-

"A further striking and at the same time more instructive proof of the equivalence of the lustre of butterfly-scales and the surface-colours of strongly absorbing dyes is to be found in the changes which the colours of these organs exhibit when immersed in fluids of varied refrangibility. 'These experiments are instructive because they disclose the manner in which the dye is contained in animal substances.

"The experiments show that, except when it is deep blue or violet, the lustre moves one or two colour-intervals in the direction from the blue towards the red end of the spectrum with increasing refrangibility of the surrounding medium, but at the same time becomes weaker. For example, the scules of Morpho menelaus, L., which glitter green-blue in air, become in ether $(n=1 \cdot 36)$ a pure green, shining less strongly, again in chloroform $(n=1 \cdot 45)$ a yellowish green and now decidedly weaker than in ether. In benzol $(n=I \cdot 52)$ and in bisulphide of carbon $(n=1 \cdot 64)$ the weak yellow-green lustre is perceptible only with direct sunshine in a dark room. In a similar manner the scales of Urania ripheus shining green in air, in ether, alcohol or water become golden yellow, the yellow red and the red blue, while in benzol and bisulphide of carbon scarcely a trace of glitter remains.

"Where we know that the canse of the lustre is a dye, the latter facts admit of but one interpretation-that in the case of butterfly-scales we have to do with solutions of the dyes in chitin, solutions whose refractivity for most of the spectrum colours is nearly equal to those of benzol and bisulphide of carbon, so that these colours, unless they are very strongly absorbed by the solution, are practically not reflected in their passage from the colourless liquids. Accordingly, the dyes which give rise to lustre in the chitin-skin of insects, and, as we shall soe presently, in the horny skin in birds, are dissolved in the same fashion as cobalt oxide in blue glass or organic dyes in a layer of solid gelatine, a conception suggested in the simple observation of the scales by transmitted light and confirmed by the facts above adduced."

If Walter's argument and conclusion are accepted, the difficulty, already considerable, of explaining the richness of the animal colours is enhanced by the supposed dilution of the dyes, and one can hardly fail to observe that a simpler

* Loc. cit. p. 96. 
explanation is to reject the dye theory and refer the colours to interference. 'The facts recorded agree pretty closely with what happens in the case of films of old decomposed glass.

Indeed, Walter, in a later passage, very candidly admits a difficulty. He says (p. 98):-

"Finally, it must not be passed over in silence that there is a circuinstance which makes a difficulty for the view here propounded of the lustre colours of butterflies. This is the fact that the lustre practically disappears in benzol and bisulphide of carbon, whereas in treating the theory of surface-colours we have several times insisted that a ray strongly absorbed must under ail circumstances be vigorously reflected."

Before leaving the question of the colours it may be well to consider an objection strongly urged by Walter against the interference theory, viz., that the colours of thin plates change too much with obliquity. As regards a single thin plate, which alone Walter seems to have contemplated, it is true, I think, that the more pronounced colours of the 2 nd and 3rd order in Newton's scale change more rapidly with the retardation* than could well be harmonized with what is observed of the animal colours. But the difficulty disappears when we admit a structure several times repeated with approximate periodicity. The changes in chlorate of potash crystals with obliquity seem to agree well enough with what is required, and this form of the interference theory has the advantage of greater elasticity, e.g. meeting Walter's objection that the colours of a single thin plate constitute a simple series with but one independent variable. Indeed, the purity of the reds often to be observed from bettles' wing-cases seems to exclude an interference theory limited to a single thin plate, inasmuch as the reds from such a plate are distinctly inferior, especially when diluted with white light reflected from an outer surface not forming part of the boundary of the thin plate $\dagger$.

Michelson, who with his great authority supports the surface-colour theory, mentions several tests under four headings (p. 561). To my mind these tests are as well, if not better, borne by an interference theory. But reliance seems to be chiefly placed upon " the more rigorous optical test of the measurement of the phase-difference and

* See a diagram of the Colours of Thin Plates, Ed. Trans. vol. xxxiii. p. 157 (1886); Scientitic Papers, vil ii. p. 498.

+ Ed. Trans. loc. cit. 
amplitude-ratios" when polarized light is reflected. I agree that this is a cogent argument, and unless it can be met the balance of evidence derived from simple observation would perhaps incline to the surface-colour theory. It is, I think, the fact that many beetles exhibit a less well-marked polarizing-angle than could be reconciled with the usual theory of thin plates constituted of non-absorbent material. An escape from the difficulty might perhaps be found in imagining a stratification composed of more than two materials, so that, for instance, the polarizing-angle for the first and second might differ considerably from that corresponding to the second and third. But such a structure seems rather improbable, and any combination of thin plates composed of two transparent materials only should give a definite polarizing-angle, abstraction being made from the minor deviations observed by Airy and Jamin.

At this point it may be recalled that a well-marked polarizing-angle and a sudden change of relative phase through two right angles are more closely connected than is sometimes realized. 'The latter without the former would involve a physical discontinuity. Michelson considers that in practice the phase-change affords the more delicate criterion *, and that in most cases it is decisive in favour of surface-colour.

A circumstance, which may perhaps be regarded as telling upon the other side is afforded by the variety of colouring at different parts, but at the same angle (e.g. at perpendicular incidence) seen in certain beetles-Dr. Hodgkinson mentions Chrysochroa fulminans. The "colours vary in an indescribable manner when attentively examined al different angles of incident light with the eye alone; with the mirror (viz., at perpendicular incidence) the wing-cases are seen to be coloured successively from base to tip iridescent green, yellow, orange, and red, and these tints remain unaltered by change of position of the object." I have confirmed generally this observation, and other beetles show something similar. The explanation makes large demands upon the surface-colour theory; but a moderate change of structure is all that would be required hy interference.

A caution is perhaps required against regarding the two theories as mutually exclusive. Both Walter and Michelson

* Some of Michelson's diagrams are rather confusing in that they sugrest a phase-difference of $180^{\circ}$ between the two polarized components reflected perpendicularly, when evidently the distinction between the two components disapperars. 
admit exceptions, and certainly there is no improbability in surface reflexion playing a part. It may be that both causes are operative in a single specimen and even at the same part of it.

The next contribution to the discussion is an important one by Vallock*, who brings to bear the instinct and experience of a naturalist as well as of a physicist. His observations were mainly on the feathers of birds and the scales of insects, and they lead him to regard interference rather than selective reflexion as the origin of the iridescent colours. "The transparency or, at any rate, the vanishing of the characteristic transmitted colour in the case of all animal tissues when immersed and permeated by a fluid of the same refractive index is strongly in favour of interferenoe being the source of the colour, but even stronger evidence is given by the behaviour of the structure under meclianical pressure.

"If' the grain or peculiarities which favour the reflexion or transmission of particular colours is of molecular size, there is no reason to suppose that pressure insufficient to cause molecular disruption would alter the action of the material on light. On the other hand, if the colours are due to interference, that is, to cavities or strata of different optical properties, compression would alter the spacing of these, and thus give rise either to different colours or, with more than a very slight compression, to the transmission and reflexion of white light."

"In every experiment of this kind that I have made either on feathers or insect scales the effect of pressure has been to destroy the colour altogether.... With many feathers the colour returns when the pressure is taken oft, but with insect scales the structure seems to be permanently injured by compression, and though when allowed to expand again the material is not colourless, the brilliancy which belonged to the uninjured scale is gone, and the colour in general changed."

"The facts above mentioned seem to offer stronger reasons in favour of interference than the polarization phenomena referred to by Michelson and Walter do against it."

I have already commented on the importance of the evidence afforded by observations with polarized light ; and if we have to choose between selective reflexion and thin plates of the type usually considered in theoretical writings, we may find ourselves in a position of much difficulty. The

* Proc. Roy. Soc. val. 1xxxr. A, p. 598 (1911). 
question then arises, Is there any loophole for escape? 1 think there may be. The polarizing angle, as given by Brewster's law, depends much upon what we may call the smoothness of the reflecting surface. A moderate curvature is of no significance in this connexion, but when the radius of curvature becomes comparable with the wavelength of the light it is another matter. 'Thus in the case of smooth glass the polarizing angle is about $57^{\circ}$-that is, light incident at this angle with the normal and vibrating in the plane of incidence is not reflected. In this observation the refected light (if there were any) would be deviated from its original direction through an angle of $2\left(90^{\circ}-57^{\circ}\right)=66^{\circ}$, and this is the direction in which light initially unpolarimed would appear completely polarized. Now replace the fiat glass by a sphere of the same material, whose diameter is small in comparison with the wave-length. Jight is now scattered in various directions, but the direction in which light originally unpolarized becomes completely polarized is at $90^{\circ}$ with the original direction, instead of $66^{\circ}$. As the sphere grows, the polarization ceases to be complete, and the direction of best polarization moves oppositely to what would be expected-that is, still further away from $66^{\circ}$. When the circumference of the sphere is equal to twice the wave-length, the polarization, still pretty good, occurs at an angle of $135^{\circ}$ with the original direction of the light*. In order to carry out the suggestion, we must abandon the supposition of uniform plame strata, inapplicable anyhow in its integrity to the case where one of the alternate plates is of air, and substitute a structure in which one of the alternatives takes a form such as the spherical. A layer of equal spheres, with centres disposed upon a plane, would give a specular reflexion and a polarizing angle dependent upon the diameter of the spheres and upon the intervals between them. In certain cases, e. $g$. when the circumference of the sphere (of glass) is equal to $1.75 \times$ wavelength, the polarization is very imperfect. To explain a brilliant and highly-coloured reflexion there would need to be several layers of spheres, and it might be supposed that the diameter varied in different layers. In this way it would seem possible to combine a specular and highlycoloured reflexion with a very imperfectly developed polarization, and thus to evade the difficulty which meets us when we confine ourselves to "thin plates." Spheres have been spoken of for simplicity and because some of

* Phil. Mag. vol. xii. p. 81 (1881) ; Proc. Roy. Soc. A. rol. 1xxxir. p. 25 (1910); Scientific Papers, vol. i. p. 518, vol. v. p. 564. 
the ffects have been calculated in this case, but it is evident that similar phenomena would be produced by obstacles of other and perhaps more probable forms. The obstucles must have a different index from that of the medium in which they are embedded, and there is no need for absorption.

It may perhaps be objected that though a layer of spheres may give a specalar reflexion there would be an accompaniment of light dispersed at other angles, forming in the case of a regular pattern "diffraction spectra." It is uncertain whether or not this occurs. If it does not, the explanation may be that the pattern is too fine.

The above remarks are intended merely to attenuate the difficulty arising from the absence of a well-marlied polarizing angle, and the detrils need not be insisted on. No surprise is felt at the deficiency of polarization in the light reflected from unpressed and unglazed paper, of which the fibres are quite large enough to be the seat of interference effects. In illustration the transverse reflexion from glass rods and fibres may be mentioned. When we examine with a nicol the reflexion from a rod $\frac{1}{4}$ inch $(6 \mathrm{~mm}$.) in diameter, we can verify the extinction at a suitable angle of the light reflected from the first surface, although abundance of other light still reaches the eye. When we replace the rod by a fine fibre, this discrimination is lost, and the rotation of the nicol may make no difference, or even a difference in the wrong direction.

The greater part of the preceding discussion was written about a year and a half ago. I am now able to supplement it with further observations of my own and of others who have been kind enough to help me. Most of my experiments have been made on wing-cases of beetles found in my garden (June and July 1917). Usually attention is first attracted by the display of a vivid green coloration, but on indoor examination the variation with angle is found to be about the same as is observed with brilliant specimens from abroad. At perpendicular incidence the colour is an orange with approach to red, passing with increasing obliquity through yellow and green to a blue-green. Ordinary solvents such as water even at the boiling-point, ether, alcohol, benzol, bisulphide of carbon, acetic acid, \&c., seem to be without effect, even when the precaution is taken to separate a wing-case into two parts so as to allow access to the interior of the cuticle. A treatment with hot caustic potash has more effect, in one experiment shifting the colour at perpendicular incidence from orange to a brilliant scarlet. By the action of hot somewhat diluted nitric acid the black 
underlying pigment may be removed without much affecting the dye, or the structure, which is the seat of the coloration.

Several experiments were made to test whether air-cavities existed. For this purpose the wing-case was exposed for some time to the action of a vacuum, into which alterwards water or benzol was admitted. But no distinct evidence of the penetration of liquid could be recorded. I understand that Prof. Poulton has had a similar experience.

Again, it has been noticed by Mr. H. Onslow and myself that considerable pressure fails to alter the colour of beetles and of the wings of some iridescent dragon-flies, though (Poulton, Mallock) effective in some other cases. It would seem that the hypothesis of air-cavities must be abandoned.

In the absence of air-cavities the alternating strneture demanded by the interference theory would require two kinds of matter capable of resisting pressure and of sensibly different refractive indices. Probably both would be solids; and since the range of relative index is then much restricted, the brightness of the reflected light could hardly be explained without supposing more than the two or three alternations which might suffice were air in question. $\mathrm{Mr}$. Onslow thinks that there may then be a difficulty in finding room for the alternating structure and the protective covering.

An important question is whether the change of colour with angle is such as can plausibly be attributed to a periodic structure. As Walter points out, a good deal depends upon whether, or not, there is a limitation upon the obliquity of the rays within the thin plate, or plates. In the ordinary arrangement for Newton's rings there is no limitation, the direction in the air-film being parallel to that of the rays before incidence upon the first plate. The optical retardation may then vary from its maximum at perpendicular incidence to zero at $90^{\circ}$ obliquity. According to this, it should always be possible to push the colour out of the spectrum at the blue end by sufficiently increasing the obliquity, but it must be remembered that unless special provision is made the colour effects would be overlaid by the white light reflected at these angles from the first glass surface encountered.

From what we have seen in the case of the beetle colours where we must suppose that the refractive index does not differ greatly from that $\left(1^{\cdot} 6\right)$ of the chitin, there is a limit to the obliquity within the thin films even when externally the incidence is grazing. If $\theta$ be the angle in the thin film and $\mu$ the refractive index, the retardation is proportional to $\cos \theta$, and in the limiting case

$$
\cos \theta=\sqrt{ }\left(\mu^{2}-1\right) / \mu .
$$


If we take $\mu=1.5$, the minimum retardation is represented by $\cdot 746$, the maximum retardation at perpendicularity being: taken as unity. It may be remarked that the minimum retardation may practically be secured without pushing very far the obliquity ontside. If we suppose the maximum retardation to give a coloration corresponding to the Fraunhofer line $\mathrm{C}(\lambda=6563)$, the minimum will correspond to $\lambda=4896$, pretty close to the line $\mathrm{F}$. According to the interference theory, then, the range of coloration should be from the full red of $\mathrm{C}$ to the blue-green of $\mathrm{F}$, and this is just about what is observed. The agreement must be admitted to be a strong argument in favour of the theory. So far as I have seen, so great a range cannot be found in the surface colour of any dye, even with the aid of polarized light.

I have already mentioned that the opaque backing behind the seat of coloration can be attacked, and for the most part removed, with nitric acid, so as to allow the transmission colour to be observed. But a much superior effect has been obtained by Dr. Eltringham, using eau de javelle (hypochlorite) *. After removal of the backing, the wing-case was mounted with balsam in a slide, which Dr. Eltringham has kindly left in my possession. Close observation of this specimen has yielded results which $I$ think interesting and tolling. Seen by transmitted light with the aid of a Coddington lens, the slide shows a pale green over the larger part of the area, which by perpendicular reflexion is a full red. The green is fairly uniform except where it appears perforated with small circular spots, which look reddish, but perhaps only by contrast. Especially to be noted is the fact that there is no colour seen by transmission at all comparable in saturation with those exhibited by reflexion. For observation of the reflected light it is advantageous, though not necessary, to renew the opaque backing, which was done by coating the under surface of the glass with gelatine darkened with ink. A good deal depends upon the source of light. In the first detailed examination, the source (a gas-mantle) happened to be highly localized, and I was puzzled to reconcile the highly spotty character of the reflexion, varving from red to green or green-blue according to the incidence, with the uniformity of the transmission tint. Similar appearances conld of course be observed in direct sunlight. But when the slide was held

* Dr. Eltringham's label ruus :-

Mimela leei. Elytron after prolmged eau-de-javelle. Only surfacefilm left. Transmits complementary colour's to those it reflects, and reflects stme colours from both sides (1917). 
very close to a large window facing a nearly uniform sky, the intervals between the spots filled up with colour, for the most part of approximately the same hue, and the reflexion was nearly uniform except for the small round holes already mentioned. Evidently the reflexion of the gas-mantle had failed to reach the eye, except from a relatively small area presenting the proper angle, thus explaining the spotty appearance observed with this illumination.

The colours reflected at moderate angles seem highly saturated. At perpendicular incidence the prism shows next to nothing beyond the uninterrupted red and redorange, and on inclination the green region appears well isolated. The impression left upon my mind is that the phenomena cannot plausibly be explained as due to surfacecolour, which in my experience is always less saturated than the transmission colour, and that, on the other hand, the interference theory presents no particular difficulty, unless it be that of finding sufficient room within the thickness of the cuticle. But the alternations cannot be those of plane strata, extending without interruption over the whole area of the colour.

As regards the difficulty of finding room sufficient for an optical structure of the kind contemplated, Mr. Onslow estimates the available thickness at from $0.75 \mu^{*}$ to $2 \mu$ in the case of many butterfly scales, and this is little enough. Even the larger estimate would amount to only about 9 or 10 half wave-lengths, even when allowance is made for the wave-length being less than in air, and the lower limit would apparently not suffice. But these measurements are not easy, and may perhaps be disturbed by refraction effects. Mr. Onslow has shown me many drawings of sections in planes perpendicular to the surface from many butterfly scales and from two or three beetle wing-cases. Most of these exhibit structures approximately periodic along the surface, but in no case a structure periodic in going inwards along the normal. But a structure of the latter kind adequate to the purpose may probably lie close upon the microscopic limit, unless, indeed, it could be made evident in a section cut very obliquely.

It must be confessed that much still remains to be effected towards a complete demonstration of the origin of these colours. Even if we admit an interference character, questions arise as to the particular manner, and there are perhaps possibilities not hitherto contemplated.

November 20, 1918.

$$
* \mu={ }_{10}^{1} 00 \mathrm{~mm}
$$

